\title{
Seleção para duração do ciclo vegetativo em batata e relação com a produtividade de tubérculos
}

\author{
Gabriel B Rodrigues'; César Augusto BP Pinto²; Flávio RG Benites ${ }^{3}$; Dheyne S Melo ${ }^{3}$ \\ ${ }^{1}$ UFV-Depto. Fitotecnia, 36571-000 Viçosa-MG; ${ }^{2}$ UFLA-Depto. Biologia, C. Postal 3037, 37200-000 Lavras-MG; ${ }^{3}$ Doutorando, UFLA- \\ Depto. Biologia; cesarbrasil@ufla.br
}

\section{RESUMO}

As cultivares de batata no Brasil geralmente apresentam ciclo vegetativo de 90 a 110 dias, sendo bem mais longo em países de clima temperado, podendo chegar até 150 dias. O ciclo vegetativo longo nestes países proporciona maior produtividade e constitui-se em alternativa para aumento da produção de tubérculos sob condições tropicais. Os objetivos deste trabalho foram avaliar o potencial da seleção para aumentar ou diminuir o ciclo vegetativo da batata e verificar a relação entre a duração do ciclo vegetativo e a produção de tubérculos em condições tropicais. Na primeira geração clonal foram avaliados a produção de tubérculos e o ciclo vegetativo de 1.561 genótipos oriundos de 22 famílias clonais. Na segunda geração clonal foram avaliadas as mesmas características em 320 genótipos selecionados para ciclo vegetativo precoce, intermediário e tardio. A seleção nas gerações iniciais foi eficiente, tanto para diminuir permitiu obter maior ganho do que a seleção entre famílias. Os genótipos mais tardios foram mais produtivos que os mais precoces.

Palavras-chave: Solanum tuberosum, precocidade.

\begin{abstract}
Selection for vegetative growth period and its relationship to tuber yield of potato

Potato cultivars in Brazil generally present a growth cycle duration ranging from 90 to 110 days, whereas in countries located in temperate climate it can reach up to 150 days. Longer vegetative growth cycle in these countries confer higher tuber yield and, in tropical countries it also could be an alternative to increase tuber yield. In this work we evaluated the selection potential for longer or shorter growth cycle duration of potato clones and we determined the relationship between growth cycle duration and tuber yield. In the first clonal generation tuber yield and growth cycle duration were evaluated on 1561 genotypes derived from 22 clonal families. In the second clonal generation, the same traits were evaluated on 320 genotypes selected for earliness, intermediate and late vegetative cycle. Selection in the initial generations was efficient both to decrease and to increase the growth cycle duration. The selection for growth cycle based on individual clones allowed higher gains than the selection among families. The latest genotypes were more productive than the earliest genotypes.
\end{abstract}

Keywords: Solanum tuberosum, earliness, cycle.

(Recebido para publicação em 22 de abril de 2008; aceito em 15 de junho de 2009)

(Received in April 22, 2008; accepted in June 15, 2009)

\begin{abstract}
$\mathrm{A}$ batata (Solanum tuberosum L.) foi domesticada na Europa a partir da seleção de clones de $S$. tuberosum ssp. andigena (ou grupo Andígena) introduzidos em 1570. O grupo Andígena é originário dos Andes, do sul do Peru e norte da Bolívia, em condições de fotoperíodo curto (cerca de 12 horas) e temperaturas amenas. Na Europa, ela foi adaptada aos dias longos do verão do norte europeu. No século XVII, ela passou a ser cultivada em diversos países no mundo (Hawkes, 1994).

No Brasil, a espécie foi introduzida no final do século XIX por imigrantes europeus. Até os dias atuais, a maioria das cultivares empregadas no país é de origem européia e sofre os efeitos adversos das temperaturas mais elevadas (Menezes et al., 2001) e do fotoperíodo mais curto. Essas condições climáticas, dentre outros fatores, contribuem para a redução do potencial produtivo das cultivares em re-
\end{abstract}

giões tropicais (Kooman \& Rabbinge, 1996).

Um dos efeitos adversos de temperaturas elevadas no comportamento da espécie é o encurtamento do ciclo vegetativo (Kooman et al., 1996). É sabido que, em regiões temperadas, as cultivares com ciclo mais longo (>130 dias) são mais produtivas que os materiais mais precoces. Silva \& Pinto (2005) demonstraram que, também para as condições tropicais, clones com ciclo mais tardio são mais produtivos que os mais precoces e sugeriram que a seleção de clones tardios constitui-se em estratégia exeqüível para aumentar a produtividade da cultura nessas regiões.

Os objetivos deste trabalho foram avaliar o potencial da seleção para aumentar ou diminuir o ciclo vegetativo da batata e verificar a relação entre ciclo vegetativo e a produção de tubérculos.

\section{MATERIAL E MÉTODOS}

Vinte e duas famílias clonais, cada qual representada por aproximadamente 100 clones, foram avaliadas neste estudo. Como testemunhas empregaram-se as cultivares comerciais Monalisa, Atlantic e Asterix, e os clones CBM 16-16 e CBM 9-10 do Programa de Melhoramento da batata da UFLA.

Dois experimentos foram implementados em condições de campo em fazendas de produção comercial. A primeira geração clonal (C-1) foi cultivada no município de Carrancas, MG (21 $1^{\circ} 35^{\prime}$ de latitude S e $44^{\circ} 53^{\prime}$ longitude $\mathrm{W}$, com $1.052 \mathrm{~m}$ de altitude), no período de 2 de fevereiro a 
2 de junho de 2006 (safra da seca). O manejo da cultura foi realizado segundo as práticas culturais usadas na região sul de Minas Gerais. Na adubação de plantio empregou-se o formulado 4-14-8 (N-P $\left.\mathrm{O}_{5}-\mathrm{K}_{2} \mathrm{O}\right)$ na dosagem de $4.000 \mathrm{~kg} \mathrm{ha}^{-1}$. Na amontoa foi realizada uma adubação de cobertura com $300 \mathrm{~kg}$ ha $^{-1}$ de sulfato de amônio. Foi utilizado o delineamento de blocos ao acaso, com quatro repetições. As 22 famílias foram distribuídas em parcelas de 25 plantas, constituindo cada planta um clone diferente, espaçados de $0,5 \mathrm{~m} \mathrm{x}$ $0,8 \mathrm{~m}$. Em cada bloco, foi incluída uma parcela com cinco testemunhas aleatorizadas, cada qual representada por cinco plantas. Avaliaram-se as características produção de tubérculos (g/planta) e o ciclo vegetativo de cada planta (clone) individualmente. Para a avaliação do ciclo vegetativo, a partir dos 78 dias após o plantio (DAP) foram realizadas sete colheitas, quando as plantas estavam com as folhas completamente secas.

Os dados foram submetidos à análise de normalidade de KolmogorovSmirnov (Steel et al., 1997) e, em seguida, realizou-se a análise de variância utilizando-se o comando PROC GLM (procedure of general linear model) do SAS (SAS, 2000).

Na primeira geração clonal estimouse a variância genética entre famílias e $\left(\sigma_{g}^{2}\right)$ a variância genética entre plantas dentro de famílias $\left(\sigma_{g d}^{2}\right)$, sendo esta obtida pela diferença da variância dentro de parcelas contendo os clones experimentais e a variância ambiental obtida nas parcelas contendo as testemunhas. Para cada característica foi estimada a herdabilidade no sentido amplo para a seleção entre famílias $\left(h_{a}^{2}\right)$ (Vencovsky \& Barriga, 1992) e a herdabilidade para a seleção de clones dentro da família $\left(h_{d}^{2}\right)$ (Paiva et al., 2002). Para cada herdabilidade, foi estimado o intervalo de confiança de acordo com Knap et al. (1985).

Foram selecionados 144 clones precoces, 122 clones intermediários e 54 clones tardios para representarem a distribuição dos clones nos diferentes ciclos, representativos de 22 famílias. Para a estimativa do ganho esperado com a seleção de três famílias $(13,6 \%)$, tanto no sentido de encurtar o ciclo vegetativo (famílias mais precoces) quanto aumen$\operatorname{tar}$ (famílias mais tardias), utilizou-se a expressão recomendada por Falconer \& Mackay (1996). O ganho esperado com a seleção dos 144 clones precoces e dos 54 clones tardios foi estimado pela mesma expressão, empregando-se como estimador da herdabilidade a expressão , $\left(h_{d}^{2}\right)$ que inclui a variância entre plantas dentro de parcelas.

$\mathrm{O}$ experimento com a segunda geração clonal (C-2) foi implementado no município de São João da Mata, MG

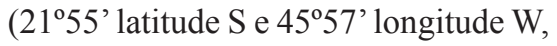
com $1.200 \mathrm{~m}$ de altitude), no período de 30 de agosto de 2005 a 12 de janeiro de 2006 (safra das águas). Os tratos culturais foram realizados conforme as práticas normalmente empregadas para a cultura no sul de Minas Gerais. A adubação de plantio foi efetuada com o formulado 4-14-8 $\left(\mathrm{N}-\mathrm{P}_{2} \mathrm{O}_{5}-\mathrm{K}_{2} \mathrm{O}\right)$ na dosagem de $3.000 \mathrm{~kg} \mathrm{ha}^{-1}$. Na amontoa, realizada aos 30 DAP, foi utilizada a mesma adubação de cobertura da primeira geração clonal.

Os 320 clones selecionados e as cinco testemunhas foram avaliados em condições de campo no delineamento de blocos ao acaso com três repetições e duas plantas por parcela no espaçamento de $0,3 \mathrm{~m} \times 0,8 \mathrm{~m}$. As parcelas das testemunhas foram aleatorizadas dentro do bloco, da mesma forma que os clones selecionados. Avaliaram-se o ciclo vegetativo por visitas semanais a partir de 74 DAP e a produção de tubérculos (g/planta) após a colheita realizada no final do ciclo vegetativo.

Os dados foram submetidos à análise de normalidade e análise de variância utilizando o comando PROC GLM (procedure of general linear model) do SAS (SAS, 2000).

Na segunda geração foi estimado o ganho realizado com a seleção de famílias, utilizando-se o comportamento médio na segunda geração dos clones das três famílias mais precoces (ou mais tardias) e o comportamento médio na segunda geração dos 320 clones selecionados. O ganho realizado com a seleção de clones precoces (ou tardios) foi estimado pelo comportamento mé- dio dos clones precoces (ou tardios) na segunda geração em relação à média geral. As herdabilidades para a seleção de famílias e para a seleção de clones foram estimadas segundo a expressão de Bernardo (2002).

As estimativas de correlação de Pearson entre as características nas duas gerações foram obtidas pelo PROC CORR (procedure correlation) do SAS (SAS, 2000), e as equações de regressão e o coeficiente de determinação, pelo Excel (Microsoft, 2002). Os pontos de máximo foram obtidos por derivação.

\section{RESULTADOS E DISCUSSÃO}

Foram obtidos dados de 1.561 clones de primeira geração, procedendo-se à classificação, se precoces $(78,85$ e 92 DAP), intermediários (99 e 106 DAP) ou tardios (113 e 120 DAP). A distribuição do número de clones que se classificaram nessas categorias foi: $46,1 \%$ de precoces, $44,7 \%$ de intermediários; e $9,2 \%$ de tardios.

Detectaram-se diferenças significativas entre as famílias e entre as testemunhas para ciclo vegetativo e produção de tubérculos na primeira geração clonal (Tabelas 1 e 2). Tanto para famílias quanto para testemunhas, $\mathrm{o}$ ciclo vegetativo foi avaliado com maior precisão que a produção de tubérculos. Pela análise de variância em nível de famílias, o coeficiente de variação ambiental foi $257,1 \%$, com a maior expressão para produção de tubérculos em relação ao ciclo vegetativo $(12,5$ versus 3,5). Tais resultados decorreram da expressiva variação no tamanho dos tubérculos-semente, que originaram as plantas da primeira geração clonal, e que, por conseguinte, impactaram fortemente a produção. Pinto et al. (1994) já haviam relatado que o tamanho do tubérculo-semente exerce um papel de destaque na produtividade do clone na geração posterior.

Nas testemunhas, os clones CBM 16-16 e CBM 9-10, que foram selecionados para condições tropicais de cultivo (Menezes et al., 2001), apresentaram ciclo vegetativo de 93,6 e 99,9 DAP, os maiores dentre as testemunhas utilizadas. Já as cultivares Atlantic, 
Tabela 1. Análise de variância para duração do ciclo vegetativo e produção de tubérculos de 22 famílias clonais de batata de primeira geração. (Analysis of variance for duration of the growth cycle and potato tuber yield for 22 clonal families in the first generation). Carrancas, UFLA, 2005.

\begin{tabular}{lccc}
\hline & GL & \multicolumn{2}{c}{ Quadrado médio } \\
\cline { 3 - 4 } & & $\begin{array}{c}\text { Ciclo vegetativo } \\
\text { (DAP) }\end{array}$ & $\begin{array}{c}\text { Produção de tubérculos } \\
\text { (g/planta) }\end{array}$ \\
\hline Bloco & 3 & 1609,1 & 468316,3 \\
Família & 21 & $977,9 * *$ & $365472,3 * *$ \\
Erro & 63 & 226,9 & 105865,8 \\
Dentro & 1687 & 67,0 & 76697,6 \\
Média & & 97,5 & 504,8 \\
\hline $\mathrm{CV}_{\mathrm{e}}^{\prime 1}$ & 3,5 & 12,5 \\
\hline$\left(\sigma_{g}^{2}\right)$ & 11,53 & 3986,10 \\
$\left(h_{a}^{2}\right)$ & 0,77 & 0,71 \\
$\mathrm{IC}_{0,05}$ & & 0,49 a 0,88 & 0,36 a 0,85 \\
$\left(\sigma_{g d}^{2}\right)$ & 35,79 & $-18575,34$ \\
$\left(h_{d}^{2}\right)$ & 0,53 & - \\
$\mathrm{IC}_{0,05}$ & & 0,35 a 0,69 & - \\
\hline
\end{tabular}

**Significativo a $1 \%$ de probabilidade pelo teste $\mathrm{F} ;{ }^{1}$ Coeficiente de variação ambiental $\left(\mathrm{CV}_{\mathrm{e}}\right)$, variância genética entre famílias $\left(\sigma_{g}^{2}\right)$, herdabilidade no sentido amplo para a seleção de famílias $\left(h_{a}^{2}\right)$ seguida do seu intervalo de confiança $\left({ }_{h_{a}^{2}}^{2}\right)$, variância genética dentro de famílias $\left(\sigma_{g d}^{2}\right)$, herdabilidade no sentido amplo para a seleção dentro de famílias $\left(h_{d}^{2}\right)$ e seu intervalo de confiança ${ }^{\left(I C_{h}{ }^{2}\right)}\left(* *\right.$ Significant at $1 \%$ level of probability by the F test; ${ }^{1}$ coefficient of environmental variation $\left(\mathrm{CV}_{\mathrm{e}}\right)$, genetic variation among families $\left(\sigma_{g}^{2}\right)$, wide sense heritability within families $\left(h_{a}^{2}\right)$ and its confidence interval $\left(I C_{h^{2}}\right)$.

Monalisa e Asterix expressaram ciclos vegetativos de 84,3, 90,4 e 91,7 DAP, respectivamente. Cumpre destacar que o ciclo vegetativo observado neste experimento foi menor do que o relatado por Silva \& Pinto (2005). Uma possível explicação para tanto foi o critério utilizado neste estudo, que considerou planta senescente aquela que contivesse apenas as folhas secas e não toda a planta seca (incluindo as hastes), como adotado por Silva \& Pinto (2005).

A variância genética para ciclo vegetativo dentro das famílias foi 3,1 vezes maior do que a variância genética entre famílias (Tabela 1). Em alguns trabalhos, tem sido constatado que as variâncias fenotípicas e genéticas são maiores dentro de famílias do que entre famílias (Bradshaw et al., 1998; Gopal, 2001; Diniz, 2006). Para a produção de tubérculos, não houve precisão ex-
As famílias mais precoces $(7,17$ e 9) apresentaram ciclos vegetativos médios de 90,6; 91,1; e 91,5 DAP; respectivamente. Já as famílias mais tardias (22, 15 e 10) tiveram, respectivamente, ciclos vegetativos médios de 105,1; 106,0; e 107,0 DAP. Para a seleção de famílias tanto para aumentar quanto para reduzir o ciclo vegetativo os ganhos estimados foram de 4,6\%. Por sua vez, para a seleção de clones precoces os ganhos preditos foram de $-11,8 \%$ e, para clones tardios, de 13,3\%. Embora a herdabilidade tenha expressado menor magnitude, há expectativa de maior ganho com a seleção para ciclo vegetativo entre clones dentro de famílias em relação às famílias. Isso, devido ao maior valor do desvio padrão genético dentro de famílias, que foi de 5,98 na comparação com 3,40 , estimado para o desvio padrão genético entre famílias. $\mathrm{Na}$ seleção entre famílias todos os indivíduos que a representam são considerados, assim, em uma família precoce, por exemplo, existem indivíduos com ciclos vegetativos precoce, intermediário e tardio. Dessa forma, a média de ciclo vegetativo de uma família é mais próxima da média geral, o que reduz o diferencial de seleção.

Dentre os 1.561 clones de primeira geração, foram selecionados 320 , de forma a representar todas as famílias em cada grupo de ciclo. A média de ciclo vegetativo dos 320 clones selecionados (97,4 DAP) foi semelhante à média dos 1.561 clones da população original (97,5 DAP). Além disso, a distribuição do número de clones em cada grupo de ciclo vegetativo revelou parecença com a constatada para os 1.561 clones, isto é, $45,0 \%$ de clones precoces, $38,1 \%$ de clones de ciclo vegetativo intermediário e $16,9 \%$ de clones tardios.

$\mathrm{Na}$ segunda geração clonal houve diferença significativa entre os genótipos, tanto para ciclo vegetativo quanto para a produção de tubérculos (Tabela 3). A média geral do ciclo vegetativo nessa geração foi de 114,2 DAP, ou seja, 16,7 dias a mais do que a média de ciclo na primeira geração clonal. Uma possível explicação para o maior ciclo vegetativo é o critério utilizado para considerar a planta como senescente, já que nesta geração considerou-se o 
Tabela 2. Análise de variância para duração do ciclo vegetativo e produção de tubérculos das testemunhas Asterix, Atlantic, CBM 9-10, CBM 16-16 e Monalisa (analysis of variance for duration of the growth cycle and potato tuber yield for the control cultivars Asterix, Atlantic, CBM 9-10, CBM 16-16 and Monalisa). Carrancas, UFLA, 2005.

\begin{tabular}{lccc}
\hline \multirow{2}{*}{ FV } & GL & \multicolumn{2}{c}{ Quadrado médio } \\
\cline { 3 - 4 } & 3 & $\begin{array}{c}\text { Ciclo vegetativo } \\
\text { (DAP) }\end{array}$ & $\begin{array}{c}\text { Produção de tubérculos } \\
\text { (g/planta) }\end{array}$ \\
\hline Bloco & 167,8 & 23151,6 \\
Testemunha & 4 & $575,6^{*}$ & $746596,0^{*}$ \\
Erro & 32 & 111,5 & 188862,4 \\
Dentro & 38 & 31,2 & 95272,9 \\
Média & & 91,9 & 644,2 \\
\hline CV $(\%)$ & & 11,5 & 67,5 \\
\hline
\end{tabular}

*Significativo a $5 \%$ de probabilidade pelo teste $\mathrm{F}$ (*significant at $5 \%$ level of probability by the F test).

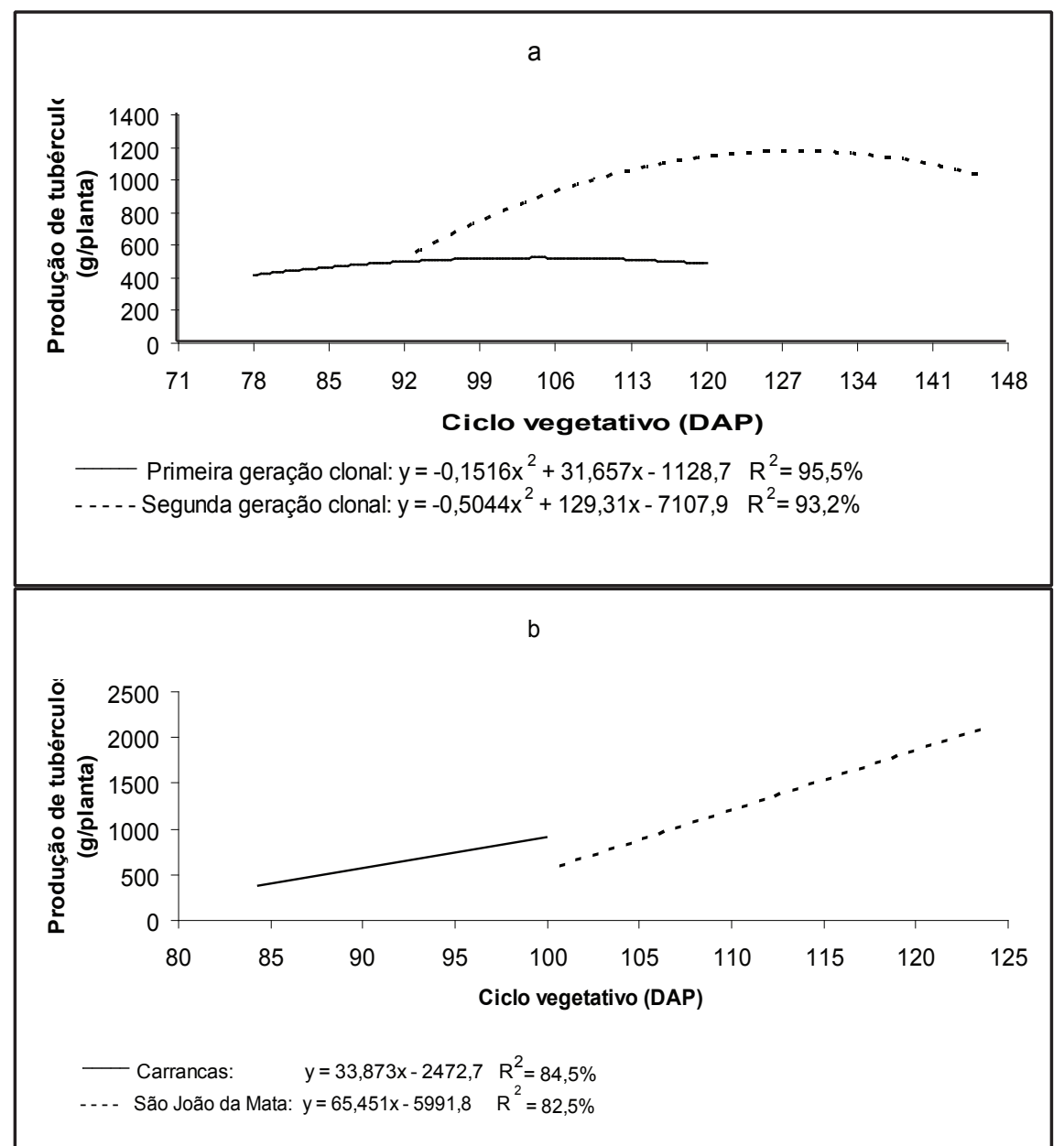

Figura 1. Produção de tubérculos em função do ciclo vegetativo na primeira e segunda geração de clones experimentais de batata (a) e de cinco testemunhas em Carrancas e São João da Mata (b) (tuber yield as a function of growth cycle in the first and second clonal generations in experimental potato clones (a) and five checks in Carrancas and São João da Mata (b)). Carrancas e São João da Mata, UFLA, 2005/2006.

término do ciclo vegetativo quando a haste estava completamente seca. Outra razão pode ter sido que as melhores condições ambientais expressas em São que tiveram rendimento $91,4 \%$ superior (1232,9 versus 644,2 g/planta). A produção média dos clones experimentais foi $100,0 \%$ superior $(504,8$ versus 1009,6 $\mathrm{g} /$ planta) àquela obtida em Carrancas. Esta superioridade reflete, além das melhores condições experimentais, o efeito do avanço da primeira para a segunda geração de seleção recorrente.

A produção de tubérculos revelou estimativas de médias menores para as cultivares (806,3 g/planta) em relação aos 320 clones selecionados (1004,9 g/ planta). Entre as testemunhas, os clones CBM 9-10 e CBM 16-16 contiveram ciclo vegetativo de 123,7 DAP e 116,3 DAP, respectivamente, os quais foram mais longos do que as cultivares Atlantic, Asterix e Monalisa, cujos ciclos foram de 100,7 DAP; 103,3 DAP; e 108,0 DAP, respectivamente. Os clones CBM 9-10 e CBM 16-16 produziram $2.370,8$ e $1.375,0 \mathrm{~g} /$ planta de tubérculos, respectivamente, enquanto Atlantic, Asterix e Monalisa produziram 845,8; 854,2; e 718,8 g/planta de tubérculos, respectivamente. Confirma-se pois a superioridade dos materiais melhorados para as condições tropicais.

De forma semelhante à primeira geração clonal, o coeficiente de variação para ciclo vegetativo na segunda geração foi de $4,8 \%$, demonstrando a boa precisão para a avaliação desta característica (Tabela 3). Contudo, para a produção de tubérculos, o coeficiente de variação foi mais elevado $(30,4 \%)$, porém, de magnitude consonante com estimativas de coeficiente de variação obtidas por Vermeer (1990) em trabalhos com batata.

As famílias 21, 3 e 4 foram as mais precoces da segunda geração clonal, ao expressarem ciclo vegetativo médio de 105,2; 107,8; e 108,6 DAP, respectivamente. Por sua vez, as famílias 11,5 e 22 foram as mais tardias, com ciclos vegetativos médios de 122,7; 124,1; e 126,9 DAP, respectivamente. Das 11 famílias mais precoces na primeira geração clonal, apenas duas não permaneceram entre as 11 famílias mais precoces da segunda geração clonal.

O ganho realizado foi de $-3,0 \%$ para a seleção de famílias precoces e de 7,0\% para as famílias tardias. No caso da seleção de clones precoces o ganho realiza- 
Tabela 3. Análise de variância para duração do ciclo vegetativo e produção de tubérculos de batata na segunda geração clonal de seleção (analysis of variance for duration of the growth cycle and potato tuber yield in the second clonal generation). São João da Mata, UFLA, 2005/2006.

\begin{tabular}{lcccc}
\hline & & & \multicolumn{2}{c}{ QM } \\
\cline { 3 - 5 } & FV & GL & $\begin{array}{c}\text { Ciclo vegetativo } \\
\text { (DAP) }\end{array}$ & $\begin{array}{c}\text { Produção de } \\
\text { tubérculos (g/planta) }\end{array}$ \\
\hline Blocos & 2 & 711,2 & 2424855,7 \\
Genótipos & 324 & $480,8^{* *}$ & $493587,0^{* *}$ \\
& Testemunhas (T) & 4 & $270,5 * *$ & $1404520,8 * *$ \\
& Clones (C) & 319 & $481,1 * *$ & $480044,2^{* *}$ \\
& T x C & 1 & $1218,3 * *$ & $1170005,2 * *$ \\
Erro & 651 & 30,0 & 93875,6 \\
Média & & 114,2 & 1009,6 \\
\hline CVe (\%) & & 4,8 & 30,4 \\
\hline
\end{tabular}

**Significativo a $1 \%$ de probabilidade pelo teste $\mathrm{F}(* *$ significant at $1 \%$ probability level by the $\mathrm{F}$ test).

Tabela 4. Correlações de Pearson entre a duração do ciclo vegetativo e a produção de tubérculos em duas gerações clonais (C-1 e C-2) (Pearson's correlation between duration of the growth cycle and tuber yield in two clonal generations (C-1 and C-2)). Carrancas e São João da Mata, UFLA, 2005/2006.

\begin{tabular}{lccc}
\hline & $\begin{array}{c}\text { Duração do ciclo } \\
\text { vegetativo } \\
(\mathbf{C}-2)\end{array}$ & $\begin{array}{c}\text { Produção de } \\
\text { tubérculos } \\
(\mathbf{C}-1)\end{array}$ & $\begin{array}{c}\text { Produção de } \\
\text { tubérculos } \\
(\mathbf{C}-2)\end{array}$ \\
\hline Duração do ciclo & $0,64^{* *}$ a & $0,30^{* *}$ & $0,20^{* *}$ \\
vegetativo (C-1) & $0,78^{* *} \mathrm{~b}$ & $0,46^{*}$ & $0,55^{*}$ \\
& $0,92^{* *} \mathrm{c}$ & $0,91^{* *}$ & $0,85^{* *}$ \\
\hline Duração do ciclo & & $0,27^{* *}$ & $0,34^{* *}$ \\
vegetativo (C-2) & & $0,42^{*}$ & $0,64^{* *}$ \\
& & $0,93^{* *}$ & $0,98^{* *}$ \\
\hline Produção de tubérculos & & & $0,32^{* *}$ \\
(C-1) & & & $0,49^{*}$ \\
& & & $0,91^{* *}$ \\
\hline
\end{tabular}

*;**significativos, a 5\% e 1\% de probabilidade, pelo teste $\mathrm{t}$; a, b, c representam, respectivamente, as correlações entre clones individuais, entre famílias e entre testemunhas $(* ; * *$ significant at $5 \%$ and $1 \%$ probability level by the $t$ test $\mathrm{a}, \mathrm{b}$ and $\mathrm{c}$ represent, respectively, correlations between individual clones, between families and between controls).

do foi de $-8,0 \%$ e, para clones tardios, de $10,0 \%$. O ganho realizado com a seleção de clones foi maior que a seleção entre famílias, confirmando a expectativa fundamentada nos ganhos esperados. A herdabilidade realizada para a seleção de famílias precoces foi de 0,50 e de 0,90 para famílias tardias, com média de 0,70 , próxima do valor estimado $\left(h_{a}^{2}\right)=$ $(0,77)$. A herdabilidade realizada para a seleção de clones precoces foi de 0,55 e de 0,48 para a seleção de clones tardios. A média dessas herdabilidade foi 0,52 e também está próxima do valor estimado $\left(h_{d}^{2}\right)=0,53$. Esses resultados denotam que a seleção para duração do ciclo vegetativo nas fases iniciais de um programa de melhoramento batata é eficiente.

As correlações entre a duração do ciclo vegetativo e a produção de tubérculos da primeira e segunda gerações clonais (Tabela 4) registram que, de modo geral, as correlações foram mais elevadas entre famílias do que entre clones individuais. A correlação entre a duração do ciclo vegetativo e a produção de tubérculos dos 320 clones selecionados na primeira geração clonal foi $\mathrm{r}=$ 0,30 (Tabela 4). Embora este valor seja menor do que $r=0,66$, estimado por Silva \& Pinto (2005), foi significativo em $p<0,01$ e, por conseguinte, denota a tendência similar de produções mais elevadas para os clones mais tardios. A correlação estimada entre clones de primeira geração provavelmente foi menor no presente trabalho em função da expressiva variação no tamanho dos tubérculos-semente, que segundo Pinto et al. (1994) afetam significativamente o desempenho dos clones na geração seguinte e que pode ter desfavorecido, parcialmente, o efeito do ciclo vegetativo mais longo. Isso fica evidenciado pela correlação positiva e significativa em $1 \%$ de probabilidade pelo teste $t$, no valor de 0,91 , entre a duração do ciclo vegetativo e a produção de tubérculos das testemunhas.

A tendência de maior produção de tubérculos para os clones com ciclo vegetativo mais longo pode ser visualizada na Figura 1. Pela equação de regressão o ponto de máxima produção de tubérculos na primeira geração clonal $(524,0 \mathrm{~g} /$ planta) ocorreu aos 104,5 DAP. A partir desse ponto, a produção de tubérculos tendeu diminuir com o alongamento do ciclo vegetativo. No que se refere às testemunhas o aumento da produção ocorreu de forma linear com o aumento do ciclo vegetativo.

A correlação entre a duração do ciclo vegetativo e a produção de tubérculos para as famílias foi mais elevada para a segunda geração do que para a primeira (Tabela 4). Provavelmente, isso se deveu ao fato de que, na segunda geração, a produtividade dos clones foi menos influenciada pelas diferenças nos tamanhos dos tubérculos-semente. Dessa forma, as diferenças no ciclo vegetativo tiveram uma participação mais acentuada na produtividade. Novamente, a estimativa da correlação entre a duração do ciclo vegetativo e a produção de tubérculos foi maior para as testemunhas, evidenciando que em gerações mais avançadas há tendência de que o efeito do ciclo vegetativo seja mais expressivo na produção de tubérculos do que 
nas gerações iniciais. Pela equação de regressão a produção máxima $(1179,7$ g/planta) ocorreu aos 128,2 DAP. Para as testemunhas o aumento da produção de tubérculos foi comparativamente de forma linearizada com o aumento do ciclo vegetativo.

Conclui-se que a seleção nas primeiras gerações clonais pode ser eficiente, tanto para diminuir quanto para aumentar o ciclo vegetativo da batata. Ademais, a seleção realizada para a duração do ciclo vegetativo entre clones possibilitou maior ganho do que a seleção entre famílias, decorrente da maior variação entre clones dentro de famílias e que os clones mais tardios tendem a ser mais produtivos que os precoces.

\section{AGRADECIMENTOS}

À CAPES pela bolsa de pós-graduação concedida ao primeiro autor.

\section{REFERÊNCIAS}

BERNADO R. 2002. Breeding for quantitative traits in plants. Minnesota: Stemma Press. $369 \mathrm{p}$.
BRADSHAW JE; DALE MFB; SWAN G; TODD D; WILSON RN. 1998. Early-generation selection between and within pair crosses in potato (Solanum tuberosum subsp. tuberosum) breeding program. Theoretical and Applied Genetics 97: 1331-1339.

DINIZ MCDR; PINTO CABP; LAMBERT ES. 2006. Sample size for family evaluation in potato breeding programs. Ciência e Agrotecnologia 30: $277-282$.

FALCONER DS; MACKAY TFC. 1996. Introduction to quantitative genetics. 4.ed. London: Longman. 464p.

GOPAL J. 2001. Between and within variation and family selection in potato breeding programs. Journal of Genetics and Breeding 36: 201-208.

HAWKES JG. 1994. Origins of cultivated potatoes and species relationships. In: BRADSHAW JE; MACKAY GR. Potato genetics. Wallingford: CAB International. p.3-42.

KNAPP SJ; STOUP WW; ROSS WM. 1985. Exact confidence intervals for heritability on a progeny mean basis. Crop Science 25: 192-194.

KOOMAN PL; RABBINGE R. 1996. An analysis of the relation between dry matter allocation to the tuber and earliness of a potato crop. Annals of Botany 77: 235-242.

KOOMAN PL; FAHEM M; TEGERA P; HAVERKORT AJ. 1996. Effects of climate on different potato genotypes 2. Dry matter allocation and duration of the growth cycle. European Journal of Agronomy 5: 207-217.

MENEZES CB; PINTO CABP; NURMBERG PL; LAMBERT ES. 2001. Combining ability of potato genotypes for cool and warm seasons in Brazil. Crop Breeding and Applied Biotechnology 1: 145-157.

MICROSOFT 2002. Project for Windows 2002, version 7: project planning software. Redmond, Wa, Microsoft Corporation. Conjunto de programas $1 \mathrm{CD}-\mathrm{ROM}$.

PAIVA JR; ALVES RE; MELO FIO. 2002. Genetic progress of selections between and within Caribbean cherry open pollination progenies. Crop Breeding and Applied Biotechnology 2: 299-306.

PINTO CABP; VALVERDE VIR; ROSSI MS. 1994. Eficiência da seleção nas primeiras gerações clonais em batata (Solanum tuberosum L.). Pesquisa Agropecuária Brasileira 29: 771-778.

SAS Institute Inc. 2000. SAS System. Cary, NC.

SILVA LAS; PINTO CABP. 2005. Duration of the growth cycle and the yield potential of potato genotypes. Crop Breeding and Applied Biotechnology 5: 20-28.

SIMMONDS NW. 1996. Family selection in plant breeding. Euphytica 90: 201-208.

STEEL RGD; TORRIE JH; DICKEY DA. 1997. Principles and procedures of statistics: A biometrical approach. 3. ed. New York: McGraw-Hill. 666p.

VENCOVSKY R; BARRIGA P. 1992. Genética biométrica no fitomelhoramento. Ribeirão Preto: SBG. 496p.

VERMEER H. 1990. Optimizing potato breeding I. The genotypic, environmental and genotypeenvironmental coefficients of variation for tuber yield and other traits in potato (Solanum tuberosum L.) under different experimental conditions. Euphytica 49: 229-239. 